\title{
Caracterização dos casos de malária na região extra amazônica brasileira entre 2012 a 2017
}

\author{
Characterization of malaria cases in the Brazilian amazon extra region \\ between 2012 and 2017
}

Caracterización de casos de malaria en la región amazónica adicional de Brasil entre 2012 y 2017

Ana Rachel Pereira BRAZ ${ }^{(1)}$

Karlla Karinne Martins Coelho BRINGEL ${ }^{(1)}$ Lorena de Albuquerque Pinheiro OLIVEIRA ${ }^{(1)}$ Israel James Cutrim de OLIVEIRA FILHO(1) Iza Luana de Oliveira TRAJANO ${ }^{(1)}$ Antônio Luís Rodrigues COSTA JÚNIOR ${ }^{(1)}$

Sueli de Souza COSTA ${ }^{(1)}$ Bruno Luciano Carneiro Alves de OLIVEIRA ${ }^{(2)}$

Recebido: 18 jun 2019 Revisado: 27 jun 2019 Aceito: 10 jan 2020

Autor de

correspondência: Ana Rachel Pereira Braz anarachelleto@hotmail.com

Conflito de interesses: Os autores declaram não haver nenhum interesse profissional ou pessoal que possa gerar conflito de interesses em relação a este manuscrito.

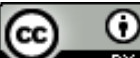

\section{Resumo}

A malária é um problema de saúde pública global, com 216 milhões de casos em 2016, sendo que no Brasil houve 191 mil em 2017. É transmitida especialmente em áreas endêmicas, na região da Amazônia brasileira, embora com registros nas áreas não endêmicas com elevada letalidade. Nesse sentido, buscou-se caracterizar o perfil epidemiológico dos portadores de malária na região extra-amazônica brasileira nos anos de 2012 a 2017 e para isso, foi realizado um estudo descritivo utilizando-se de dados secundários dos casos confirmados e notificados de malária na região extra-Amazônica brasileira, obtidos dos arquivos no Sistema de Informação de Agravos de Notificação. No período em questão, foram notificados 3.797 casos, a maioria homens (79,0\%), de 20-39 anos de idade (48\%) e 40-59 anos de idade (35,5\%), raça não branca $(46,6 \%)$, na região sudeste do país e em decorrência $P$. vivax $(66,7 \%)$, e cujos primeiros sintomas surgiram nos meses de dezembro a março. Observou-se perfil importante de características associadas aos casos na área não endêmica do país e que ações de controle e vigilância permanentes precisam ser realizadas contra o mosquito transmissor da doença.

Descritores: Malária; Epidemiologia; Saúde Pública; Doenças Transmissíveis. 


\begin{abstract}
Malaria is a global public health problem, with 216 million cases in 2016, and in Brazil there were 191 thousand in 2017. It is transmitted especially in endemic areas, in the Brazilian Amazon region, although with records in non-endemic areas with higher lethality. In this sense, we sought to characterize the epidemiological profile of malaria patients in the extra-Amazonian region of Brazil between 2012 and 2017, and for this, a descriptive study was conducted using secondary data from confirmed and reported cases of malaria in the extra-Amazon region Brazil, obtained from the files in the SINAN Notification of Injury Information System. In the period, 3,797 cases were reported, the majority of which were male (79.0\%), 20-39 years old (48\%) and 40-59 years old (35.5\%), no white $46.6 \%$, with a more prevalent $P$. vivax parasite result (66.7\%), and whose first symptoms appeared in the months of December to March. It was observed an important profile of characteristics associated with the cases in the non-endemic area of the country, and that permanent control and surveillance actions must be carried out against the mosquito transmitting the disease.
\end{abstract}

Keywords: Malaria; Epidemiology; Public Health; Communicable Diseases.

\begin{abstract}
Resumen
La malaria es un problema mundial de salud pública, con 216 millones casos en 2016, y en Brasil hubo 191.000 en 2017. Se transmite especialmente en zonas endémicas, en la región amazónica brasileña, aunque con registros en zonas no endémicas con alta letalidad. En este sentido, se trató de caracterizar el perfil epidemiológico de los portadores de malaria en la región brasileña extraamazônica en los años 2012 a 2017 y para ello, se realizó un estudio descriptivo utilizando datos secundarios de casos confirmados y La malaria reportada en la región brasileña extra-amazônica, obtenida de los expedientes del sistema de información sobre enfermedades notificables. En el período en cuestión, se notificaron 3.797 casos, en su mayoría hombres (79,0\%), 20-39 años de edad (48\%) y 40-59 años de edad (35,5\%), raza no blanca (46,6\%), en la región sureste del país y como resultado de $P$. vivax $(66,7 \%)$, y cuyos primeros síntomas surgieron en los meses de diciembre a marzo. Se observó un importante Perfil de características asociadas a los casos en la zona no endémica del país y que las acciones de control y vigilancia permanentes deben realizarse contra el mosquito que transmite la enfermedad.
\end{abstract}

Palabras-claves: Malaria; Epidemiología; Salud Pública; Enfermedades Transmisibles.

\title{
Introdução
}

A malária é uma doença causada por protozoários do gênero Plasmodium, ${ }^{1}$ e se constitui um problema de saúde pública global. ${ }^{2}$ Segundo o último relatório da Organização Mundial de Saúde - OMS, o World Malaria Report 2017, foram estimados 216 milhões de casos de malária em 91 países em 2016, cinco milhões a mais que no ano de 2015. ${ }^{3}$ A OMS, preocupada com o impacto econômico e social em vários países, tem adotado normas para eliminação e controle da doença. ${ }^{4,5}$ Apesar disto, no Brasil, em 2017, foram registrados cerca de 191.000 casos da enfermidade, ${ }^{6}$ sendo que em 2013 foram confirmados 178.546 casos, $^{2}$ e, em 2012, o Brasil era responsável por 54 \% dos casos de malária nas Américas, ${ }^{2}$ denotando preocupante quadro epidemiológico, sendo necessário que se compreenda a epidemiologia 
desta doença, de forma temporal e espacial, a fim de que seja possível o planejamento de estratégias de controle e eliminação da doença em todo o país. ${ }^{7,8}$

A parasitose é transmitida aos seres humanos pela picada de vetores - fêmeas infectadas de mosquitos do gênero Anopheles, ${ }^{1,2,9}$ que se alimentam de sangue para a nutrição de seus ovos e os depositam na água; devido a este fato, em alguns locais, a transmissão é mais intensa em períodos durante e após estações de chuva.,10 $\mathrm{O}$ ciclo de vida do mosquito dura em média 30 dias, embora algumas espécies possam alcançar até 100 dias, dependendo de fatores como temperatura e umidade do $\operatorname{ar}^{2}$ As duas espécies de plasmódios associados à doença mais distribuídas são a $P$. falciparum, principalmente no continente africano e $P$. vivax, predominante em regiões tropicais e subtropicais. ${ }^{11}$

A OMS tem promovido esforços na adoção de normas para eliminação e controle da doença, baseadas em evidências, visando reduzir a inaceitável carga da malária e seu impacto econômico e social em diversos países. ${ }^{1}$ Dentre as localidades consideradas endêmicas, destaca-se a região Amazônica brasileira (Amazônia Legal), que compreende os estados do Maranhão, Mato Grosso e os da região Norte (Acre, Amazonas, Amapá, Pará, Rondônia, Roraima e Tocantins). ${ }^{12}$ Estudo realizado em 2010 afirma que $50 \%$ dos casos registrados encontravam-se nos estados do Amazonas, Rondônia e Pará. ${ }^{13}$ Isso se deve majoritariamente às condições ambientais como vegetação, umidade e temperatura, que colaboram para a presença dos vetores, ao desmatamento e ao desequilíbrio ecológico causado pela intervenção humana. ${ }^{6,14}$ Além disso, as condições socioeconômicas da área também favorecem a transmissão da doença, uma vez que há uma maior exposição aos fatores de risco e dificuldade na adoção de medidas preventivas e de tratamento. ${ }^{15}$

Por outro lado, apesar da região amazônica deter mais de 99\% dos casos de malária no país, ${ }^{7,16}$ é possível observar também a existência de registros nas regiões não endêmicas ${ }^{11}$ sejam estes autóctones ou importados tanto da Amazônia, como de países da América Latina e da África. ${ }^{17}$ A problemática reside em uma maior susceptibilidade às formas graves da doença por dois motivos principais: baixa resistência imunológica dos indivíduos ao Plasmodium, uma vez que essa se desenvolve ao longo do tempo de exposição ao patógeno ${ }^{15}$ e dificuldade por parte dos profissionais de saúde em realizarem o diagnóstico correto, seja por falta de experiência ou pela imprecisão da sintomatologia apresentada. $^{16,17,18}$

A epidemiologia da malária na Amazônia Legal tem sido mais estudada ao longo dos anos, porém a doença ocorre disseminada fora desta região, inclusive em estados mais

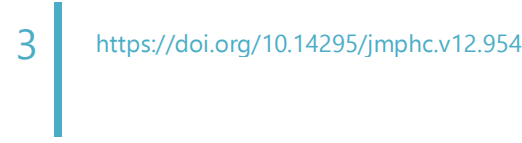


urbanizados e industrializados, ${ }^{8}$ sendo escassos os estudos que descrevem sua característica de ocorrência na área extra-amazônica.

Assim, é necessário também focar a atenção para fora da região endêmica do país, que compreende 17 estados e o Distrito Federal, visando compreender a evolução e disseminação da doença, a fim de maior controle sobre a mesma. Tendo em vista essa necessidade, o objetivo do presente estudo foi caracterizar o perfil epidemiológico dos casos de malária na região extra-Amazônica brasileira entre os anos de 2012 a 2017.

\section{Metodologia}

Trata-se de estudo descritivo dos casos confirmados e notificados de malária na região extra-Amazônica brasileira, no período de 2012 a 2017. Os dados secundários foram obtidos dos arquivos on line disponíveis no Sistema de Informação de Agravos de Notificação - SINAN, que reúne informações de doenças de notificação compulsória, e compõe o sítio virtual do DATASUS.

As variáveis selecionadas foram: ano de ocorrência, Unidade da Federação (UF) de notificação, sexo, faixa etária (em anos), cor/raça, escolaridade, região do país, resultado do parasitológico e mês do primeiro sintoma. Foram selecionadas apenas as UF de notificação não pertencentes à Amazônia Legal, ou seja, região extra-amazônica brasileira.

Os dados obtidos a partir do SINAN foram tabulados utilizando-se o programa Microsoft Excel 2016. Nas análises estatísticas, foram realizadas frequências absolutas, relativas e as taxas brutas de casos confirmados por 100.000 habitantes de acordo com a UF de cada ano no período entre 2012 a 2017.

Este estudo foi realizado exclusivamente com dados secundários, de domínio público, disponíveis no SINAN, e obedeceu às diretrizes sobre pesquisa envolvendo seres humanos recomendadas pelo Conselho Nacional de Saúde na Resolução CNS n 466, de 12/12/2012.

\section{Resultados e Discussão}

Na região extra-amazônica de 2012 a 2017 foram notificados 3.797 casos de Malária. Predominaram casos em pessoas do sexo masculino $(79,0 \%)$. As faixas etárias mais atingidas foram $20-39$ anos (48\%) e 40-59 anos (35,5\%) representando $83,5 \%$ dos casos. A raça branca foi a mais atingida pela doença (43,3\%), seguida da parda (34,3\%).

A escolaridade foi ignorada na maioria das notificações $(31,7 \%)$ e $19,3 \%$ tinham escolaridade ensino médio completo representando o grupo mais afetado. $\mathrm{O}$ resultado

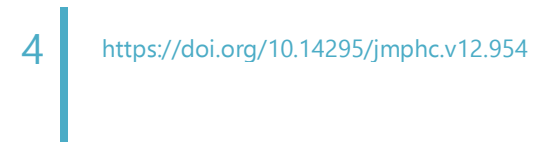


parasitológico $P$. vivax foi o mais prevalente $(66,7 \%)$. A distribuição de casos por mês de aparecimento do primeiro sintoma apresentou-se aproximadamente bem distribuída, com um leve predomínio dos meses de dezembro a março, sendo os mais frequentes janeiro $(11,9 \%)$ e fevereiro $(9,9 \%)$ (Tabela 1$)$.

Tabela 1. Caracterização dos casos de Malária $(n=3.797)$ na região extra-amazônica brasileira, entre 2012 a 2017

\begin{tabular}{|c|c|c|}
\hline Variável & $\mathrm{N}$ & $\%$ \\
\hline \multicolumn{3}{|l|}{ Sexo } \\
\hline Masculino & 2.999 & $79,00 \%$ \\
\hline Feminino & 798 & $21,00 \%$ \\
\hline \multicolumn{3}{|l|}{ Faixa etária (em anos) } \\
\hline$<20$ & 298 & $7,90 \%$ \\
\hline 20-39 & 1.824 & $48,00 \%$ \\
\hline $40-59$ & 1.347 & $35,50 \%$ \\
\hline$\geq 60$ & 328 & $9,60 \%$ \\
\hline \multicolumn{3}{|l|}{ Cor/Raça } \\
\hline Branca & 1.634 & $43,00 \%$ \\
\hline Não branca & 1.769 & $46,60 \%$ \\
\hline Ignorado & 394 & $10,40 \%$ \\
\hline \multicolumn{3}{|l|}{ Escolaridade } \\
\hline Analfabeto & 26 & $0,70 \%$ \\
\hline Fundamental Incompleto & 637 & $16,80 \%$ \\
\hline Fundamental Completo & 505 & $13,30 \%$ \\
\hline Médio Completo & 732 & $19,30 \%$ \\
\hline Superior Completo & 602 & $15,90 \%$ \\
\hline Ignorado & 1.295 & $33,10 \%$ \\
\hline \multicolumn{3}{|l|}{ Resultado do parasitológico* } \\
\hline Falciparum & 1.104 & $31,40 \%$ \\
\hline Vivax & 2.343 & $66,80 \%$ \\
\hline Malariae & 28 & $0,80 \%$ \\
\hline Ovale & 35 & $1,00 \%$ \\
\hline \multicolumn{3}{|l|}{ Mês do primeiro sintoma } \\
\hline Janeiro & 453 & $11,90 \%$ \\
\hline Fevereiro & 374 & $9,90 \%$ \\
\hline Março & 333 & $8,80 \%$ \\
\hline
\end{tabular}




\begin{tabular}{|lcc}
\hline \multicolumn{1}{c}{ Variável } & $\mathrm{N}$ & $\%$ \\
\hline Abril & 278 & $7,30 \%$ \\
Maio & 319 & $8,40 \%$ \\
Junho & 267 & $7,00 \%$ \\
Julho & 340 & $9,00 \%$ \\
Agosto & 274 & $7,20 \%$ \\
Setembro & 280 & $7,40 \%$ \\
Outubro & 274 & $7,20 \%$ \\
Novembro & 276 & $7,30 \%$ \\
Dezembro & 329 & $8,70 \%$ \\
\hline
\end{tabular}

Fonte: Elaborado pelos autores (2019).

*Foram excluídos todos os resultados com associação de espécies de Plasmodium e com presença de gametócitos de mais de uma espécie $(n=287)$.

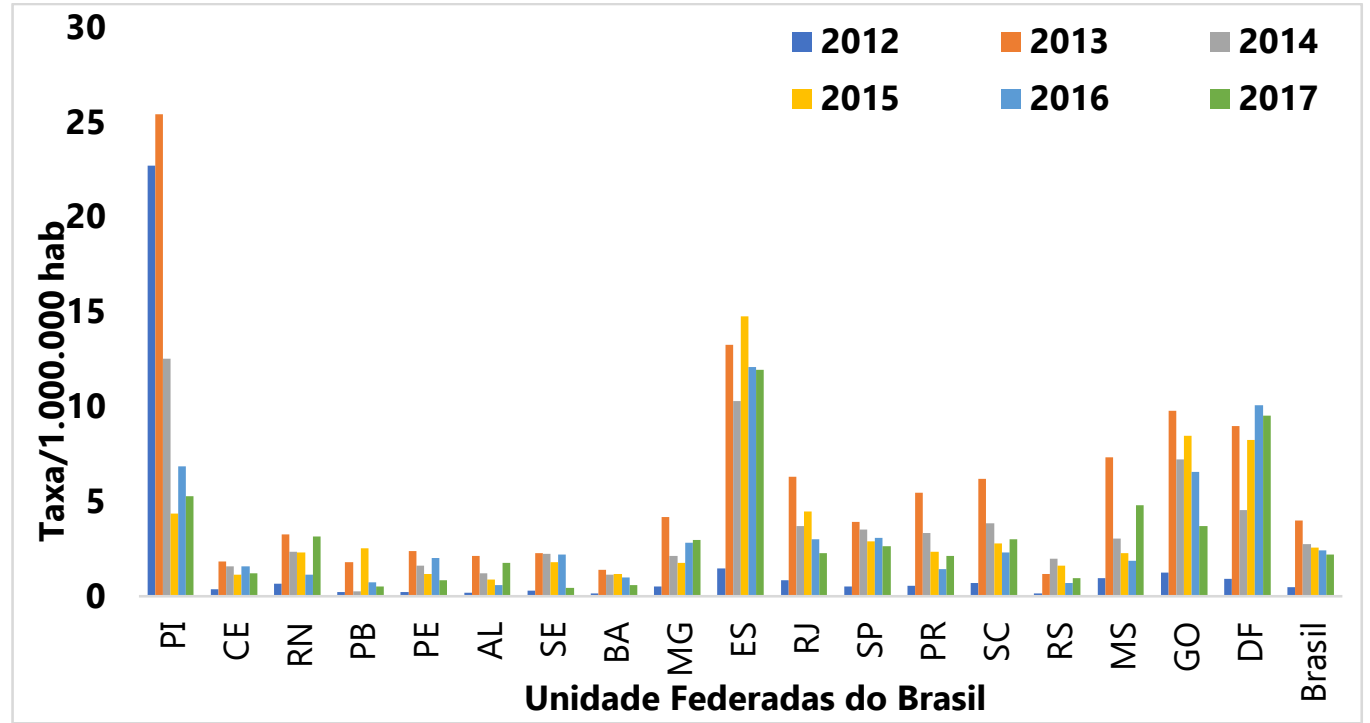

Gráfico 1. Taxa de casos confirmados de Malária na região extra-amazônica segundo a unidade de federação e o ano de Notificação, Brasil, entre 2012 a 2017

Fonte: Elaborado pelos autores (2019).

O Gráfico 2 mostra a proporção de casos de malária notificados de acordo com a UF de notificação. Os estados da Região Sudeste se mostraram com maior prevalência, sendo o estado de São Paulo o que apresentou a maior proporção (24,0\%) seguido do estado do Rio de Janeiro (12,0\%) e em Minas Gerais (10,0\%), juntos representaram 46,0\% casos. Para todas as faixas etárias, o sexo masculino teve maior proporção de casos. Observou-se que as faixa etárias de $20-39$ (38,7\% masculino e 9,3\% feminino) e de $40-59$ anos (28,6\% 
masculino e 6,9\% feminino) representam as maiores proporções de casos de malária (Gráfico 3).

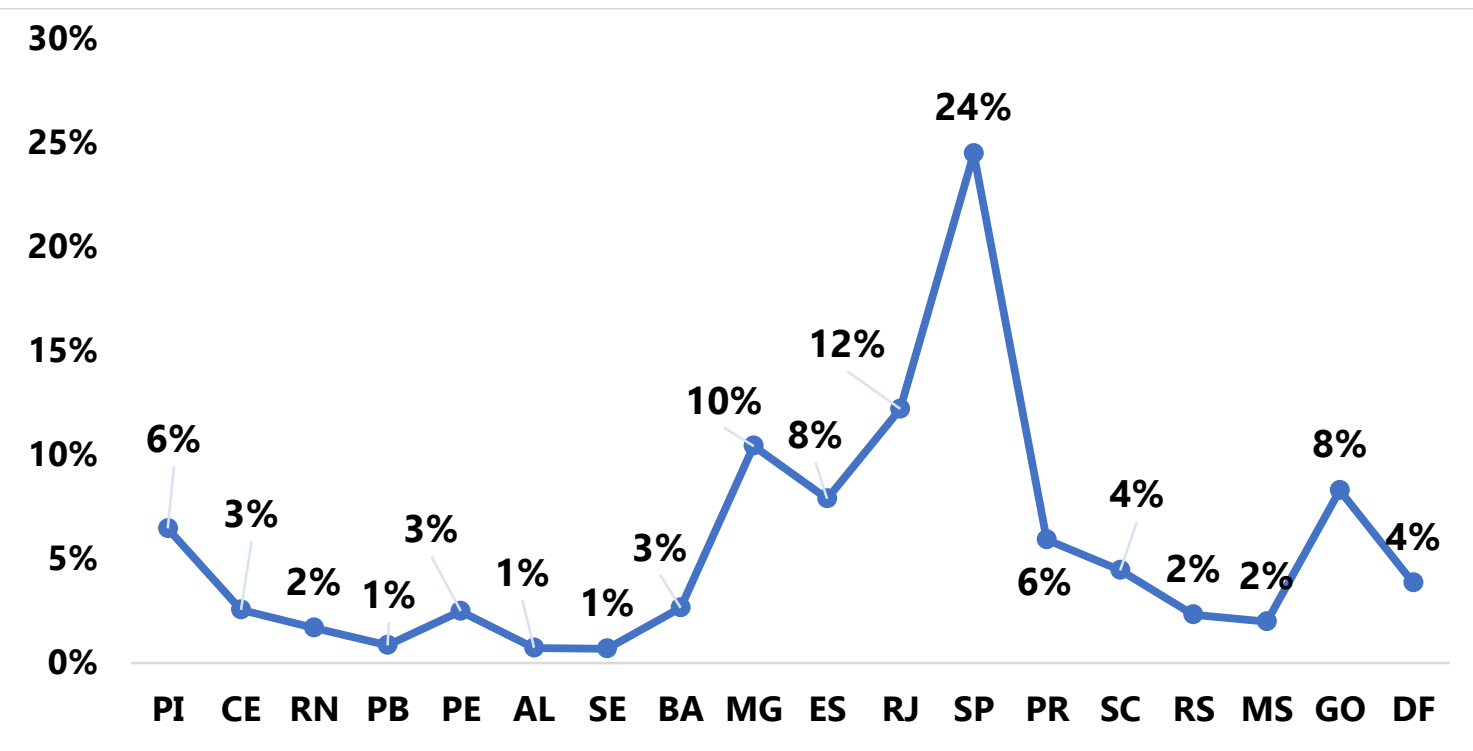

Gráfico 2. Proporção de casos confirmados $(n=3.797)$ de Malária na região extra-amazônica brasileira segundo a unidade de federação de Notificação, Brasil, entre 2012 a 2017.

Fonte: Elaborado pelos autores (2019).

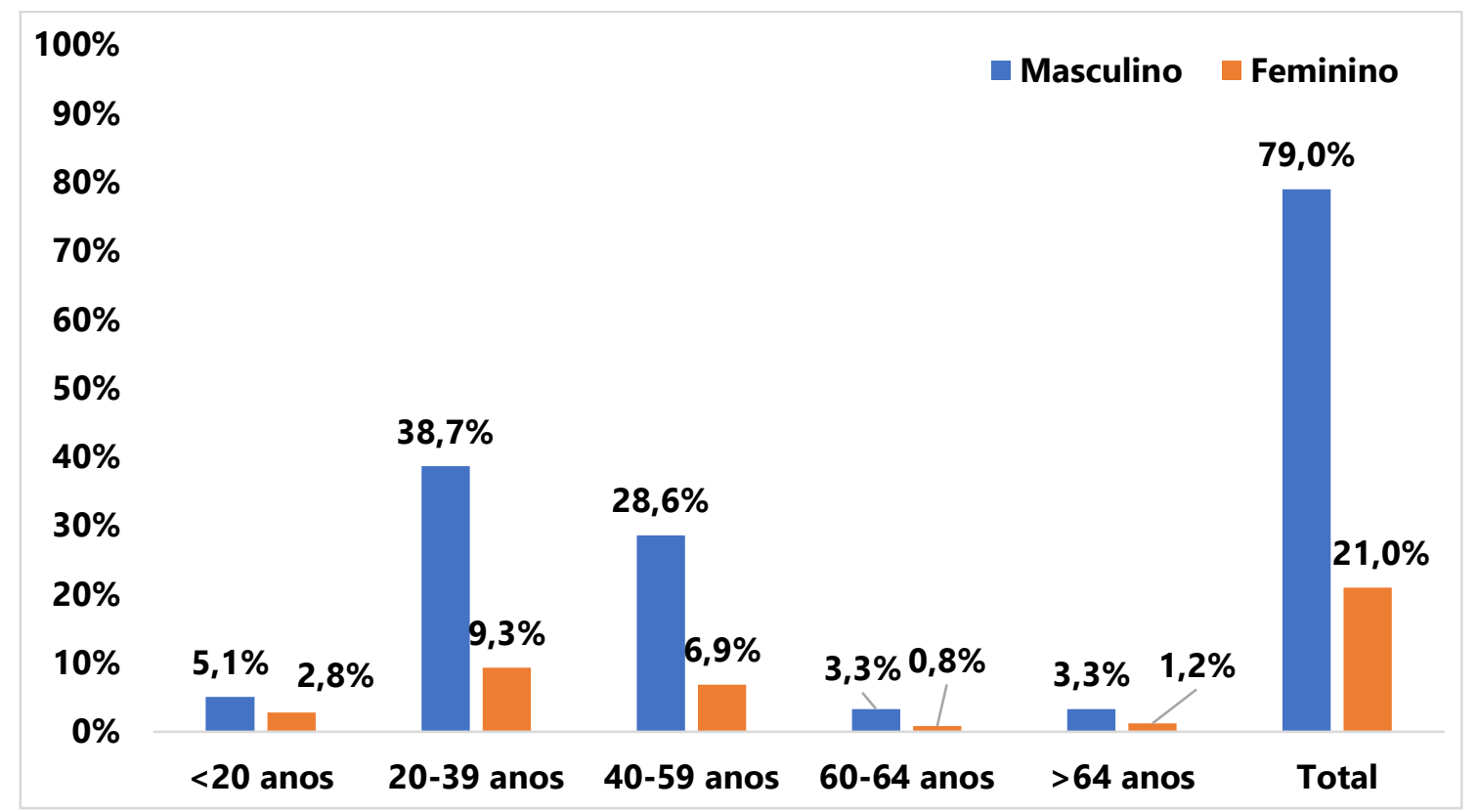

Gráfico 3. Proporção de casos notificações $(n=3.797)$ de Malária na região extra-amazônica segundo sexo e a faixa etária, Brasil, entre 2012 a 2017.

Fonte: Elaborado pelos autores (2019).

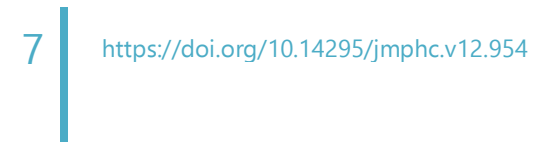


A região Nordeste apresentou maior proporção de notificação de casos de malária nos meses de janeiro (12,5\%) e dezembro (10,3\%). Na região Sudeste, os meses de janeiro $(11,9 \%)$ e fevereiro $(10,5 \%)$ representam maior proporção de notificações. Para a região Sul, os meses de janeiro $(13,3 \%)$ e dezembro $(11,3 \%)$ foram os meses com maior proporção de notificações. Na região Centro-Oeste os meses de fevereiro (11,0\%) e Janeiro $(9,9 \%)$ e a nível nacional, janeiro $(11,8 \%)$ e fevereiro $(9,8 \%)$ apresentaram as maiores proporções de notificação de Malária, mostrando assim há maior proporção de aparecimento da doença na região extra-amazônica durante os meses de dezembro, janeiro e fevereiro, que são os meses do verão para o hemisfério sul e de intensas chuvas (Gráfico 4).

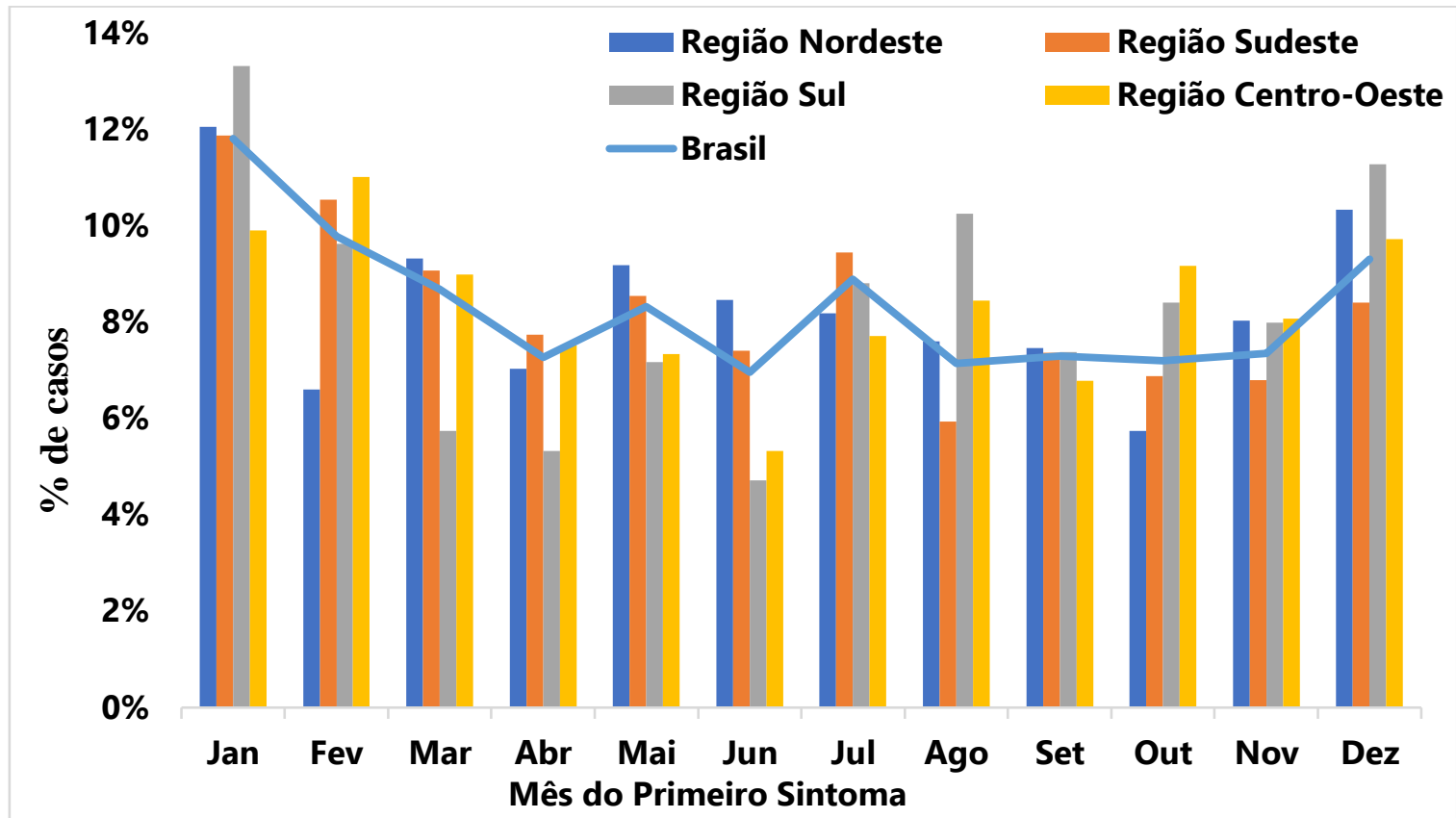

Gráfico 4. Proporção de casos notificação de Malária na região extra-amazônica brasileira segundo o Mês do Primeiro Sintoma e da região do Brasil, entre 2012 a 2017.

Fonte: Elaborado pelos autores (2019).

\section{Discussão}

Os resultados do estudo apontam perfil importante de características associadas a ocorrência de malária fora da região amazônica brasileira, indicando que os casos foram mais frequentes entre homens, adultos jovens, de cor/raça não branca, de baixa escolaridade, em decorrência do Plasmodium vivax e com o aparecimento dos primeiros sintomas nos meses de dezembro a março, que representam o período sazonal de chuvas e de férias na estação de verão. A proporção e as taxas de incidência de malária vêm reduzindo

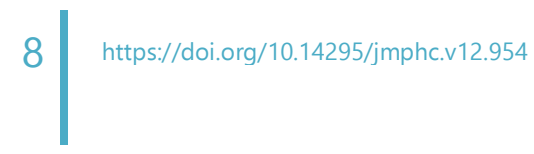


ao longo dos anos, mas foram maiores no estado Piauí e estados da região sudeste e centrooeste.

A malária atinge cerca de $40 \%$ da população do planeta, porém com incidência maior nas regiões tropicais e subtropicais. ${ }^{7,19}$ Dados do Datasus apontam que entre 2007-2012, houve um total de 6.092 casos registrados nas regiões extra-amazônicas, determinando, portanto, uma diminuição no número de notificações entre 2012 e 2017 (3.797 casos). Até 1995, a incidência de casos de malária no país era elevada, com dados apontando, como causa, a ocupação e colonização da região amazônica, principalmente.

Entretanto, desde 2000, foram traçadas metas para a sua redução, com a implantação do Plano de Intensificação das Ações de Controle da Malária, pelo governo federal, havendo a descentralização das ações de vigilância em saúde e a mobilização de vários setores do Ministério da Saúde para o controle da doença. ${ }^{20} \mathrm{Em} \mathrm{2010}$, foi criado o Programa Nacional de Controle da Malária, que, além de contribuir para reduzir a carga pela doença, foi lançado no intuito de também prevenir complicações que poderiam levar a morte, visando ainda eliminar a transmissão de áreas urbanas e mantém a ausência da doença em locais onde já teria sido interrompida, utilizando como estratégias o diagnóstico precoce e de qualidade, e o tratamento especifico, oportuno e de ótima qualidade, além do controle do mosquito transmissor, especialmente na região endêmica. ${ }^{20} \mathrm{O}$ programa destaca o fato de que "nas regiões em que a malária não é endêmica, tem-se observado manifestações graves da doença, possivelmente pelo retardo da suspeita clínica, do diagnóstico e do tratamento", ${ }^{20, \text { p. } 9}$ chamando a atenção para os sintomas e a necessidade de tratamento imediato, e sendo desta forma necessário o delineamento do perfil da doença fora da região endêmica.

Corroborando com a literatura, os dados encontrados no presente estudo apontam que o perfil epidemiológico da malária na região extra-Amazônica nos anos de 2012 a 2017 foi predominado por indivíduos do sexo masculino. ${ }^{21,22}$ Essa maior prevalência nesse sexo (78,98\%), se deve principalmente ao fato de que as áreas mais favoráveis para o desenvolvimento do vetor, como na agricultura, garimpo, pesca e exploração de madeira, estão associadas na maioria das vezes às atividades realizadas pela mão de obra masculina, aumentando a exposição ao mosquito Anopheles. ${ }^{21,22}$ A transmissão da doença depende da atividade exercida, podendo ser de caráter extra ou peridomiciliar, sendo que as mulheres tendem a permanecer mais tempo no interior das residências, e por isso menos expostas à picada dos insetos transmissores. ${ }^{11}$ Além disto, os achados deste trabalho vão ao encontro dos dados obtidos em estudos anteriores, sendo que no período de 2005 a 2009 a 
distribuição dos casos de malária por sexo foi de $70 \%$ para sexo masculino e $30 \%$ para o sexo feminino. ${ }^{23}$

Quanto à faixa etária predominante (20-39 anos), esta é a mesma prevalência encontrada em estudos realizados em Sergipe, ${ }^{24}$ na região do baixo Amazonas, ${ }^{25}$ e no Rio Grande do Sul, ${ }^{26}$ três regiões completamente distantes geograficamente e com características diferentes do ponto de vista sócio-ambiental. Essa maior prevalência nesse grupo está associada ao fato de serem a população mais exposta a atividades de trabalho externo.

No presente estudo, entre os 3.797 (100\%) casos de malária ocorridos na região extraamazônica no período de estudo, tem-se como resultados parasitológicos predominantes a espécie $P$. vivax. Na literatura existente é traçado o mesmo perfil com variações mais frequentes de $P$. vivax $^{13,14}$ e menor de $P$. falciparum ${ }^{14}$ mantendo, semelhança com os resultados deste estudo.

Embora o Piauí seja considerado como área livre da transmissão da doença desde meados da década de $1985,9,27$ ao serem analisadas a presença de casos notificados naquele estado, entre 2002 e 2013, utilizando o Sistema de Distribuição Geográfico - SIG padrão de distribuição espaço-temporal dos surtos de casos de malária está concentrado na porção noroeste do estado, na fronteira com o Maranhão. No período 2002-2012, no Maranhão, foram confirmados 73.425 casos da doença. ${ }^{21}$

Além disto, entre 2007-2012, houve um total de 6092 casos registrados nas regiões extra-amazônicas, transformando-se num fato preocupante, pois essas áreas altamente povoadas mantêm condições favoráveis à disseminação dos parasitas e vetores. ${ }^{8}$ Fora da região Amazônica, os estados de São Paulo, Minas Gerais, Rio de Janeiro e Espírito Santo são os que apresentam maior número de notificações da doença, ${ }^{12}$ tendo sido notificados 499 casos de malária extra-amazônica entre janeiro e outubro de $2017{ }^{28}$ A letalidade da malária na região extra-amazônica é cerca de 80 vezes maior do que na Amazônia, principalmente devido ao diagnóstico tardio, ${ }^{8,29,30}$ pois apenas $19 \%$ destes casos têm diagnosticado e tratamento nas primeiras 48 horas após o início dos sintomas, em contraste com $60 \%$ dos casos de malária na região amazônica. ${ }^{8}$

Na região extra-Amazônica, proporcionalmente em relação ao número de casos de malária notificados, o predomínio é no Sudeste; sendo os estados de São Paulo, Rio de Janeiro e Espírito Santo que contam respectivamente com 24\%, 12\% e 8\% dos casos, e fazem parte da Mata Atlântica - área cujas condições ambientais promovem meio adequado para o desenvolvimento dos mosquitos vetores da doença., ${ }^{7,16} \mathrm{Na}$ década de 1950, a malária 
nessas localidades tornou-se conhecida como malária de bromélia, uma vez que essas plantas permitem o acúmulo de água; atualmente, os casos autóctones são considerados residuais, porém ainda são estatisticamente relevantes. ${ }^{16,30,31}$

Os meses que mostram maior número de notificações da patogenia são dezembro, janeiro e fevereiro. No Brasil, esse período, nos anos de 2013 a 2017, de acordo com as informações disponibilizadas pelo Instituto Nacional de Meteorologia - INMET, há limites pluviométricos e de temperatura superiores às médias estabelecidas, ${ }^{32}$ facilitando o acúmulo de água doce, que permite o estabelecimento do ciclo de vida do Plasmodium, juntamente com as temperaturas mais elevadas, que aceleram o desenvolvimento das fases do parasita, contribuindo para a disseminação da doença. ${ }^{16,30,31}$

Dentre as limitações desse estudo estão o elevado número de dados ignorados para importantes variáveis como escolaridade e cor/raça. Além disso, o subregistro de casos pode limitar nossa compreensão sobre o verdadeiro cenário epidemiológico associado a essa doença. Entretanto, profissionais de saúde devem estar atentos, também na região extraamazônica, quanto aos sinais e sintomas da malária, especialmente nas regiões onde a doença tem aparecido com maior frequência, e nos meses em que há maior quantidade de chuvas, com temperatura elevada.

\section{Conclusão}

Os resultados apontaram que há volume importante de casos de malária ocorrendo na área não endêmica do Brasil e que esses casos indicaram perfil de características socioeconômicas, demográficas e climáticas associadas ao agravo. Novos estudos devem ser realizados, caracterizando a progressão/diminuição de casos na região não-endêmica, a fim de nortear as ações de controle em vigilância e saúde permanentes precisam ser realizadas contra o mosquito transmissor da doença.

\section{Referências}

1. Rey L. Bases da parasitologia médica. $3^{\text {a }}$ ed. Rio de Janeiro: Guanabara Koogan; 2011.

2. Braz RM, Tauil PL, Santelli ACFS, Fontes CJF. Avaliação da completude e da oportunidade das notificações de malária na Amazônia Brasileira, 2003-2012. Epidemiol Serv Saude. 2016;25(1):21-32. http://dx.doi.org/10.5123/s1679-49742016000100003. 
3. World Health Organisation. World malaria report 2017. Geneva; 2017. Disponível em: https://www.who.int/malaria/publications/world-malaria-report-2017/en/

4. D'Souza BJ, Newman RD. Strengthening the policy setting process for global malaria control and elimination. Malar J. 2012 Jan 27;11:28. https://doi.org/10.1186/1475-2875-11-28.

5. Alonso $\mathrm{PL}$, Brown $\mathrm{G}$, Arevalo-Herrera $\mathrm{M}$, et al. A research agenda to underpin malaria eradication. PLoS Med. 2011 Jan;8(1):e1000406. https://doi.org/10.1371/journal.pmed.1000406.

6. Ferreira TDC. Caracterização e análise dos fatores de risco da transmissão da malária na Amazônia Legal, 2010-2015: uma contribuição à saúde global [tese]. [São Paulo]: Universidade de São Paulo; 2018. http://dx.doi.org/10.11606/T.6.2018.tde-11062018-102152.

7. Costa AP, Bressan CS, Pedro RS, et al. Diagnóstico tardio de malária em área endêmica de dengue na extra-Amazônia Brasileira: experiência recente de uma unidade sentinela no estado do Rio de Janeiro. Rev Soc Bras Med Trop. 2010;43(5):571-4. http://dx.doi.org/10.1590/S0037-86822010000500020.

8. Lorenz C, Virginio F, Aguiar BS, et al. Spatial and temporal epidemiology of malaria in extraAmazonian regions of Brazil. Malar J. 2015 Oct 15;14:408. https://doi.org/10.1186/s12936-0150934-6.

9. Santos AC, Valladares GS, Hassum IC. Distribuição espaço-temporal da malária no Piauí 2002-2013. Hygeia Ver Bras Geogr. 2015 Dez [acesso 6 maio 2019:11(21):1-19. Disponível em: http://www.seer.ufu.br/index.php/hygeia/article/view/28942.

10. World Health Organization. Malaria [Homepage]. [s.I.]: WHO; 2018 [acesso 16 ago. 2018]. Disponível em: http://www.who.int/news-room/fact-sheets/detail/malaria.

11. Mesquita EM, Muniz TF, Sousa ALS, et al. Levantamento epidemiológico da malária no estado do Maranhão, Brasil nos anos de 2007 a 2012. Rev Cienc Saude. 2013[acesso 16 ago. 2018];15(1):11-8. Disponível em: http://www.periodicoseletronicos.ufma.br/index.php/rcisaude/article/view/1917

12. Gomes AP, Vitorino RR, Mendes $T A$, et al. $A$ infecção pelo gênero Plasmodium: epidemiologia, profilaxia e controle no Brasil. Vittalle. 2018 [acesso 6 maio 2019]:30(2):4858. Disponível em: https://periodicos.furg.br/vittalle/article/view/7472/5401. 
13. Braz RM, Andreozzi VL, Kale PL. Detecção precoce de epidemias de malária no Brasil: uma proposta de automação. Epidemiol Serv Saude. 2006 jun;15(2):21-33. http://dx.doi.org/10.5123/S1679-49742006000200004.

14. Lapouble OMM, Santelli ACFS, Muniz-Junqueira MI. Situação epidemiológica da malária na região amazônica brasileira, 2003 a 2012. Rev Panam Salud Publica. 2015[acesso 16 ago. 2018];38(4):300-6. Disponível em: https://scielosp.org/pdf/rpsp/2015.v38n4/300-306/pt

15. Braz RM, Duarte EC, Tauil PL. Caracterização das epidemias de malária nos municípios da Amazônia Brasileira em 2010. Cad Saude Publica. 2013;29(5):935-44. http://dx.doi.org/10.1590/S0102-311X2013000500011.

16. Brasil $\mathrm{P}$, Costa $\mathrm{AP}$, Longo $\mathrm{CL}$, et al. Malaria, a difficult diagnosis in a febrile patient with submicroscopic parasitaemia and polyclonal lymphocyte activation outside the endemic region, in Brazil. Malar J. 2013 Nov 7;12:402. http://dx.doi.org/10.1186/1475-2875-12-402.

17. Oliveira MRF, Giozza SP, Peixoto HM, et al. Cost-effectiveness of diagnostic for malária in Extra-Amazon Region, Brazil. Malar J. 2012 Nov 23;11:390. http://dx.doi.org/10.1186/14752875-11-390.

18. Dotrário $A B$, Menon $L B$, Bollela VR, et al. Malaria and other febrile diseases among travellers: the experience of a reference centre located outside the Brazilian Amazon Region. Malar J. 2016 May 26;15(1):294. http://dx.doi.org/10.1186/s12936-016-1347-x.

19. Oliveira-Filho AB, Martinelli JM. Casos Notificados no Estado do Pará, Amazônia Brasileira, de 1998 a 2006. Epidemiol Serv Saúde. 2009;18(3):277-84. http://dx.doi.org/10.5123/S167949742009000300010.

20. Ministério da Saúde (BR). Guia prático de tratamento da malária no Brasil. Brasília, DF: 2010. 36 p. (Série A. Normas e Manuais Técnicos). Disponível em: http://bvsms.saude.gov.br/bvs/publicacoes/guia_pratico_malaria.pdf

21. Aguiar LC, Batalha ADP, Silva RBL. A malária no estado do Maranhão: casos notificados de 2002 A 2012. Rev Pesqui Saude.2014 [acesso 6 maio 2019];15(3):346-50. Disponível em: http://www.periodicoseletronicos.ufma.br/index.php/revistahuufma/article/view/3657/1657.

22. Freitas FDS. Caracterização epidemiológica dos casos de malária no município de Icatu-MA [monografia]. [São Luís, MA]: Faculdade Santa Terezinha; 2010. $63 \mathrm{f}$. 
23. Lopes NFSN, Tadei WP, Brito LMO, et al. Malária no Maranhão: análise dos fatores relacionados com a transmissão no período de 2005 a 2009. Rev Pesqui Saude. 2013 [acesso 2 fev. 2018];14(1):40-4. Disponível em:

http://www.periodicoseletronicos.ufma.br/index.php/revistahuufma/article/view/1723

24. Alves Neto JS, Cruz ARC, Melo MCA, et al. Malária em região extra-Amazônica: perfil dos casos atendidos em hospital de referência de Sergipe. Sempesq. 2016;(18):1-2. Disponível em: http://www.sbmt.org.br/medtrop2016/wp-content/uploads/2016/12/8693-

Mala\%CC\%81 ria-em-regia\%CC\%83o-extra-Amazo\%CC\%82nica-perfil-dos-casos-atendidosem-hospital-de-refere\%CC\%82ncia-de-Sergipe.pdf.

25. Sousa JR, Santos ACF, Almeida WS, et al. Situação da malária na Região do Baixo Amazonas, Estado do Pará, Brasil, de 2009 a 2013: um enfoque epidemiológico. Rev Panamazonica Saude. 2015;6(4):39-47. http://dx.doi.org/10.5123/S2176-62232015000400006.

26. Moreira, DL. Aspectos epidemiológicos da malária no Rio Grande do Sul [dissertação]. [Canoas, RS]: Unilasalle; 2017.

27. Chagas FB, Araújo TME, Santos AC, et al. Aspectos epidemiológicos e entomológicos de malária autóctone no município de Uruçuí - Piauí - Brasil [CD-ROM]. XLIX Congresso da Sociedade Brasileira de Medicina Tropical; 2013 ago.; Campo Grande, MS: SBMT; 2013.

28. Ministério da Saúde, Secretaria de Vigilância em Saúde, Sistema de Informação de Vigilância Epidemiológica da Malária SIVEP-Malária. Situação epidemiológica da malária na região Amazônica, período de 2015 a 2017 [acesso 2 fev. 2018]. Disponível em: https://public.tableau.com/profile/mal.ria.brasil\#!/vizhome/MiniSivep1517_2018_01_04/caso s_notifi cados_2017_regio_Amazonica.

29. Braz RM, Tauil PL, Santelli ACFS, et al. Avaliação da completude e da oportunidade das notificações de malária na Amazônia Brasileira, 2003-2012. Epidemiol Serv Saude. 2016;25(1):21-32. http://dx.doi.org/10.5123/s1679-49742016000100003.

30. Pina-Costa A, Brasil P, Di Santi SM, et al. Malaria in Brazil: what happens outside the Amazonian endemic region. Mem Inst Oswaldo Cruz. 2014;109(5):618-33. http://dx.doi.org/10.1590/0074-0276140228.

31. Sousa, ARM. Estudo da dinâmica de transmissão de malária autóctone de Mata Atlântica: análise da variação na acrodendrofilia de Anopheles cruzii (Diptera: Culicidae) e desenvolvimento de modelo matemático para a transmissão zoonótica [tese]. [São Paulo]:

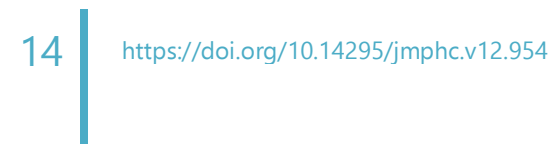


Faculdade de Saúde Pública; 2018. http://dx.doi.org/10.11606/T.6.2018.tde-27092018144306.

32. Instituto Nacional de Meteorologia. Previsão Sazonal do INMET [Homepage]. [São Paulo: INMET; 2018 [acesso 16 ago. 2018]. Disponível em: http://www.inmet.gov.br/portal/index.php?r=clima/prev_estocastica.

\section{Minicurrículo}

Ana Rachel Pereira Braz | ORCiD: 0000-0002-8847-5793

Acadêmica do curso de medicina da Universidade Federal do Maranhão - UFMA, Campus em Pinheiro, MA, Brasil

Karlla Karinne Martins Coelho Bringel | ORCiD: 0000-0001-7812-9857

Acadêmica do curso de medicina da Universidade Federal do Maranhão - UFMA, Pinheiro, MA, Brasil.

Lorena de Albuquerque Pinheiro Oliveira | ORCiD: 0000-0002-7658-8416

Acadêmica do curso de medicina da Universidade Federal do Maranhão - UFMA, Pinheiro, MA, Brasil.

Israel James Cutrim de Oliveira Filho | ORCiD: 0000-0003-1873-2157

Acadêmico do curso de medicina da Universidade Federal do Maranhão - UFMA, Pinheiro, MA, Brasil.

Iza Luana de Oliveira Trajano | ORCiD: 0000-0001-7906-7280

Acadêmica do curso de medicina da Universidade Federal do Maranhão - UFMA, Pinheiro, MA, Brasil.

Antônio Luís Rodrigues Costa Júnior | ORCiD: 0000-0003-1165-2293

Nutricionista. Mestrado em Saúde Coletiva. Docente do curso de medicina da Universidade Federal do Maranhão - UFMA, Campus em Pinheiro, MA, Brasil.

Sueli de Souza Costa | ORCiD: 0000-0003-4127-7324

Cirurgiã-dentista. Doutorado em Ciências Odontológicas. Docente do curso de medicina da Universidade Federal do Maranhão - UFMA, Pinheiro, MA, Brasil.

Bruno Luciano Carneiro Alves de Oliveira | ORCiD: 0000-0001-8053-7972

Enfermeiro. Doutorado em Saúde Coletiva. Docente do curso de medicina da Universidade Federal do Maranhão - UFMA, Pinheiro, MA, Brasil e do Programa de Pós-graduação em Saúde Coletiva - PPGSC - UFMA. 